\section{THE COX-2 PATHWAY AS A MEDIATOR OF RESISTANCE TO ANTI-PD-1 THERAPY}

Shuming Chen*, Tracee McMiller, Preethi Sankaran, Kyle Kampta, Suzanne Topalian. Johns Hopkins University School of Medicine, Baltimore, MD, USA

Background We previously found upregulation of the cyclooxygenase-2/prostaglandin E2 (COX-2/PGE2) pathway in the tumor microenvironment (TME) of cancers that respond poorly to anti-PD-1 therapy. ${ }^{1-2}$ The potential functional role of this pathway in anti-PD-1 resistance is unknown. We therefore studied modulation of COX-2 expression in cultured human tumor and immune cells, PGE2-mediated effects on myeloid cells and their reversal with prostaglandin (EP) receptor inhibitors.

Methods Nineteen tumor lines representing 6 histologies were treated with cytokines reported to induce COX-2 (IL-1B, IL17A, TNF-a). Peripheral blood monocytes (Monos) were treated with toll-like receptor (TLR) agonists or TME-resident cytokines associated with high PD-L1 expression (IL-1A, IL10, IL-27, IL-32g, IFN-g). ${ }^{3-4}$ COX-2 protein was detected by Western blotting and flow cytometry. In some experiments, Monos were pre-incubated with EP2i (PF-04418948) and/or EP4i (ONO-AE3-208), then treated with PGE2 \pm TLR4 (LPS) or TLR7 (imiquimod) agonists. IL-6, IL-10, TNF-a, and VEGF secretion were detected by ELISA. Monocytic DCs generated with GM-CSF+IL-4 were matured with CD40L, \pm PGE2, then phenotyped.

Results Among 19 tumor cell lines, 6 expressed COX-2 constitutively, and 13 were induced to express COX- 2 by 1-day exposure to IL-1B, IL-17A, or TNF-a. In Monos, COX-2 was induced by IL-1A and IL-1B, but not IFN-g or IL-27. TLR 19 agonists induced COX-2, with TLR2/4/5 agonists being the strongest inducers. COX-2 induction by these factors was nonoverlapping with PD-L1 induction in tumor cells and Monos, suggesting non-redundant pathways of immune resistance. PGE2 had context-dependent effects in Monos, depending on the cytokines, TLR agonists, and donors assayed: PGE2 increased VEGF secretion by resting Monos from 4/4 donors tested, but increased IL-6, IL-10 and TNF-a secretion in only 1/4 donors; PGE2 increased imiquimod-induced TNF-a secretion, but decreased LPS-induced TNF-a secretion. EP2 and EP4 inhibitors counteracted PGE2-mediated cytokine modulation, and showed synergistic effects when combined in the context of high dose of PGE2 (500nM). Additionally, PGE2 suppressed the in vitro generation of mature DCs, reducing CD80 and CD83 expression and increasing CD16.

Conclusions Understanding and preventing anti-PD-1 treatment resistance is a critical goal. Our results suggest that the COX2/PGE2 pathway is expressed in tumor and immune cells, and modulates myeloid cell functions in a context-dependent manner. COX-2 expression is non-redundant with PD-L1 expression, providing a rationale to test COX-2 pathway inhibition in conjunction with anti-PD-1. Available drugs targeting this pathway, including IL-1R and IL-1B inhibitors, NSAIDs, and EP2 and EP4 inhibitors, will enable the clinical development of combination treatment regimens.

Acknowledgements We gratefully acknowledge support from NCI R01-CA142779, Bristol Myers Squibb, and the Johns Hopkins Bloomberg Kimmel Institute for Cancer Immunotherapy.

\section{REFERENCES}

1.. Duffield AS, Ascierto ML, Anders RA, et al. The immunosuppressive tumor microenvironment (TME) in nasopharyngeal carcinoma: implications for immunotherapy. AACR 2018;Abstract 4750.

2.. Besharati $\mathrm{S}$, McMiller $\mathrm{T}$, Yarchoan $\mathrm{M}$, et al. The immunosuppressive tumor microenvironment (TME) in epstein-barr virus (EBV)-positive and EBV-negative gastric cancers: implications for immunotherapy. SITC 2018;P541 (abstr).

3.. Taube JM, Young GD, McMiller TL, et al. Differential expression of immune-regulatory genes associated with PD-L1 display in melanoma: implications for PD-1 pathway blockade. Clin Cancer Res 2015:21:3969-76.

4.. Duffield AS, Ascierto ML, Anders RA, et al. Th17 immune microenvironment in epstein-barr virus negative Hodgkin lymphoma: implications for immunotherapy. Blood Advances 2017;1:1324-34.

http://dx.doi.org/10.1136/jitc-2021-SITC2021.288 